\title{
Research and Reflection on the "Low Rating but High Grossing" Phenomenon in Chinese Commercial Films: A Case Study of Detective Chinatown 3
}

\begin{abstract}
Ziyi Zhou
Fujian Normal University, Communication, Fuzhou, Fujian, 350100

Email:brown0913@163.com

ABSTRACT

This paper focuses on the "low rating but high grossing" phenomenon in recent years' movies. Faced with the contradiction between high box office and the immature development of commercial films, how to improve film brands has become the main object of Chinese film marketing in the future. In response to the current problems, this paper proposes some paths to construct film brands: firstly, clarify the mind of a film, and complete high-concept scripts through market research and consumer positioning; secondly, play the role of behavior identity of director brands and star brands by adopting a comprehensive media strategy; furthermore, emphasize the development of visual identity derivatives and upgrade film brand consumption in the process of extending the industrial value; in addition, utilize "cultural field" with mature IP to help build better film brands.
\end{abstract}

Keywords: commercial film, film brand, marketing strategy, film industry.

\section{BACKGROUND}

With the accelerating of the overall scale of film industry, the declining and rising of new audience groups has also brought about the evolution of consumption patterns. New media has made film marketing the most flexible and malleable part of film industry and will play an important role in the ultimate realization of the industry's market value. Among the thriving market-oriented films, the much-anticipated New Year movie Detective Chinatown 3 before its release this year has so far won the box office of 4.522 billion yuan, ranking fifth in the box office list in Mainland China. However, after the first round of rating, the film fell flat on all major platforms. Many disappointed viewers even gave low scores, making the film the representative of this year's "low-rating but high-grossing" movies.

With respect to the difficult coordination between film industrialization and artistic connotation, and the film content which reduces the public's aesthetic taste and spiritual realm, this paper aims to analyze the causes of "low rating but high grossing" phenomenon, further discuss the marketing strategies and brand construction of the Chinese film industry, and reexamine the relationship between domestic marketing methods and the film industry with the help of the mature Hollywood industry system, so as to explore the development direction of Chinese commercial films.

\section{LITERATURE REVIEW}

There are few studies on the "low-rating but high-grossing" phenomenon in China, mainly published in periodicals and magazines, and the research objects are contemporary and individually different. In recent years, as film marketing, one of the important parts of film industry, has gradually received the industry's attention, many scholars have set their sights on it and continue to expand and deepen related research. With respect to this, many scholars only focus on the macro-market level, and only a small number of them conduct research from the micro-level perspective.

\subsection{Relevant Research on the "Low-rating but High-grossing” Films}

For "low-rating but high-grossing" films or so-called lousy movies, the academic circles have not given a precise definition. Professor Li Tianyi believes that lousy movies are a kind of lowlevel aesthetic taste[1]; Associate Professor Zheng Yiyong indicates that the most distinctive sign of a lousy film is that its plot is too simple or the story is confusing, which cannot bear closer analysis[2]. From the perspective of consumer psychology and the market, Wang Chujing analyzed the causes of this phenomenon from three aspects: the upgrading of the industry pattern, the change of film-watching motivation, and the rise of film marketing[3]. Hu Yihao mentions the audiences' psychology of curiosity and the ugly towards entertainment in 
the Internet era in the article "The Surging Path of and Reflection on the Negative Review Marketing of Chinese Films"[4].

\subsection{Relevant Research on Marketing Activities of Commercial Films}

In the early years, some scholars have put forward suggestions on film marketing in China. Yao Rui[5], Liu Fan, and Liu Jingya[6] all stated that video marketing, twitter marketing, and WeChat marketing based on new media are the mainstream marketing methods, but these methods are too simple to be applied to the current marketing environment.

However, in recent years, in domestic scholars' research on Western film marketing strategies, Su Xingsha summarized the Contemporary Hollywood Film Industry as the state of the American film industry from the 1980 s to the present, including the labor and star system of the film industry, intellectual property rights, and Hollywood products of the global market and various new media[7]; $\mathrm{Hu}$ Yun, from the perspective of the film industry, believes that being a large multinational film entertainment company lies in the vertical integration of the industry and the horizontal expansion of mergers and integration across industries[8], which still provides references for commercial film marketing in China.

\section{DILEMMA AND CAUSES OF "LOW RATING BUT HIGH GROSSING" PHENOMENON: A CASE STUDY OF DETECTIVE CHINATOWN 3}

\subsection{Dilemma and contradiction: the conflict between business spirit and artistic connotation}

Films are originally a kind of spiritual culture, and should pay more attention to spiritual value than business earnings. However, the success of movies in modern era is measured by box office, and entertainment and industrialization have become the mainstream. Hou Li Youmei put forward the postmodern image spectacle and criticized the current phenomenon of "rising box office, degenerate films", thinking that films have shifted from "connotative narrative" to "spectacle display"[9]. In the era of films for mass entertainment, this phenomenon not only shows the result of excessive commercialization, but also indicates people's lack of spiritual culture. Faced with thriving market-oriented box office revenue, seeking the harmony between market's business and cultural attributes, and the unity of rational humanistic thinking and consumption should be the thinking direction of current commercial films.

\subsection{Causes}

\subsubsection{Underdeveloped industrial structure}

The traditional film industry generally includes four stages: financing, production, release, and screening. Film marketing starts from the production stage and reaches its climax before screening. Detective Chinatown 3, through multi-media layout, all-platform joint and integrated marketing, multi-channel material distribution, event marketing, cross-media promotion, etc., surpassed the ceiling of the pre-sale box office with 717 million yuan. Compared with Western films whose operation model is highly integrated, China's commercial films in marketing are basically limited to film screening. For instance, Detective Chinatown 3 has almost no follow-up publicity and post-film industry development.

The development of the entire industrial chain requires a complete industrial system and a sound industrial scale. The Chinese film industry chain is still immature and cannot reach Western standards in the short term. China's commercial film market relies on box office revenue from theaters. Obtaining substantial box office through comprehensive integrated marketing has become the main direction of the marketing of China's film industry. For film distributors, the premise is to ensure box office revenue, but they have ignored word-ofmouth evaluation and follow-up marketing after the release, as well as the value extension through the development of derivatives.

\subsubsection{Brand Effect of Series of Films}

Film industrialization stimulates the industry to construct brands and reap high box office through brand effect and brand influence. According to the systematic segmentation of commercial film brands, currently representative film brands in China include director brands, celebrity brands, and series of films, among which the series of films are the reproduction of films, and the consolidation and expansion of film brands. As Zhang Weiping, chairman of Beijing New Picture Film Co., Ltd. once said: “A film's success is a prerequisite for producing film brand effect; and good film brand effect will promote each other with social effect and jointly consolidate the success of the film. [10]"

Compared with new films' competition in the content, Detective Chinatown 3's competition stands at a new point. The success of the first two series of Detective Chinatown and its derivative TV series is a prerequisite for the film Detective Chinatown 3, the only live-action film brand for the Spring Festival in 2021, to grab the audiences' attention.

The familiar cast of director Chen Sicheng and lead actors Wang Baoqiang and Liu Haoran, and strong base of audience contribute to film's number one position in ticket sales. As for the film's subsequent decline in reputation, the main reason is that the film's "style" is out of touch with that of the previous series. Compared with the first two films which largely grabs people's attention through "suspense", the third film greatly weakens the reasoning suspense part but strengthens the "comedy" part. Professor Hou Keming also believes that the film is hollow and flat in characterization; and mixed and composite genres make its style difficult to grasp[11]. Besides, the film also lacks aesthetic value and blended in storylines. All of these reduced its actual value and provided the basis for audiences' low rating. 


\subsubsection{Sinking consumption and evolving market}

Previous film creators tend to reflect and polish on the artistic, ideological, and social natures of films, and concentrate on the films' content itself. However, in the development of the film industry and the reference of the foreign film industry market, current films present a consumer-oriented trend. The emergence of commercial films allows market research and consumer analyses to be integrated into film production. What's more, from a viewer's perspective, more and more people prefer to seek spiritual comfort by relaxing and entertaining in the cinema due to the pressure of life. Therefore, films have gradually transformed from an artistic expression into a tool for emotional venting and relaxation. As a family-friendly New Year movie, Detective Chinatown 3 strengthens the dramatic effect of "comedy", but does not have too much in-depth content related to the meaning of life, the value of existence, and the attention to social issues. On the one hand, it has gained a high box office through catering to the masses and displaying in a burden-free viewing mode; on the other hand, it has also received low ratings because of its dull content and simple plot.

\section{MARKETING STRATEGY OF CHINESE SERIES OF FILMS BASED ON CIS IMAGE RECOGNITION SYSTEM: A CASE STUDY OF DETECTIVE CHINATOWN 3}

Though Detective Chinatown 3's reputation has declined, considering the box office alone, it is still a successful work. Leaving aside objective external factors such as consumption patterns, market orientation, and theaters, the internal factors of the film's success come from its brand effect. In the Detective Chinatown series, even the name of the film plays an important role, distinguishing it from other films. From the perspective of the construction of the CIS brand image recognition system in branding, the construction of a film brand includes three aspects: (1) "Mind Identity" (MI for short): value norms and cultural concepts that are recognized and abided by, namely, the film style, which is the basic feature of genre films; (2) "Behavior Identity" (BI): behavioral expression of film concepts, and various production and operation behaviors, mainly manifested as the relationship between actors and role images, marketing behaviors; (3) "Visual Identity" (VI): visual expression, including mise-en-scene, color tone, light and shadow, soundtrack, editing and other shooting techniques and characteristics of the form of expression, as well as the development of post-film products with film characteristics.

Generally speaking, throughout the process, series of films have unified "behavior", "mind" and "identity" that can be easily recognized by consumers. These films can also continuously consolidate the audience base during the viewing process, making them loyal consumers of the film brand.

In the process of continuous accumulation and strengthening of a film brand's series, the style of directors and actors has gradually become the "behavior trademark" of the film and helps form a differentiated film brand that can be identified. The visual identity system of the series of films also complements the expression of film element symbols. The narrative rhythm, classic lines, core props, and scenes are separated from the film itself and become special symbols. In the development of a series of films, the addition of symbols familiar to the audience in the previous films can often arouse the favor of loyal consumers and help them form a positive consumption attitude. In this way, special symbols that come down in a continuous line contribute to people's positive association about the film brands. They become the asset of the film brands, and at the same time, create possibility of film "materialization" and realize the film brands' commercial value.

\section{REFLECTION AND LEARNING: DILEMMA OF CHINESE FILM MARKETING VIEWING FROM THE INDUSTRY MODEL OF MARKETING OF HOLLYWOOD SERIES OF FILMS}

\subsection{Reference of the Marketing Strategies of Hollywood Series of Films}

\subsubsection{Script creation with market significance: "high-concept" commercial films}

Generally speaking, "high-concept" commercial films adopt film story models that focus on the audience market, employ a brisk and easy-to-understand narrative manner to expand the scope of promotion, and promote the main lineup with commercial attributes to the audience. Such films have been invested a lot of money. They pursue high returns with high investment. Since the 21 st century, Hollywood has launched many phenomenal series of commercial films which have formed a wide range of responses around the world, such as the Harry Potter series and the Lord of the Rings series. These "high-concept" series of films feature in simple dialogue, grand scenes and special effects, popular storylines, and modular character design, thus influence the global market.

\subsubsection{Strategic adjustment of market focus:} Paying more attention to the development of derivatives and extend the industrial value chain

As the cost of films increases year by year, the box office is no longer the only way to gain profits since box office revenue alone cannot make up for investment losses. Therefore, in addition to box office, the revenue of derivatives, DVD sales, and paid media platforms often take a lion's share of the total. Compared with short-term and fast-growing benefits in the theatres, Hollywood movie company giants pay more attention to 
follow-up long-term income in the audio and video stores and entertainment market, since the post-film products that can be continuously discovered have their unique commercial value. Therefore, the development of film elements, from simple peripheral products to theme parks or other products within a larger scope, provides the possibility for the development of series of works.

\subsection{Extension and development}

It is well recognized that there is no shortage of excellent script IPs in the literary fields such as modern Chinese novels and historical masterpieces. These excellent works possess a high reputation and spreading range, but most of them have not formed a complete and unified series. Therefore, the adaptation of well-known works can harvest a large number of potential audience groups, and can also form an interaction force with the original works, and achieve the "cultural field" of value development $\quad 1+1>2$ through multi-party cooperation[12]]. What's more, unique high-quality IP can form a variety of variants in the development of derivatives, recreate and reproduce the subject, thus create a classic IP image with content value and commercial value.

\section{CONCLUSION}

The "low rating but high grossing" phenomenon sounds an alarm for the prospect of Chinese commercial films. In the information age, the marketing form of commercial films cannot standstill at a large-scale online and offline integrated marketing model. However, thinking about how to achieve the compatibility between artistic value and commercial value, and creating scripts based on market analyses in the early stage and realizing the development of post-film industry in the later stage are the future development directions of Chinese film marketing. At the same time, the adaptation of excellent script IPs should also become the future consideration direction of Chinese commercial films.

\section{REFERENCES}

[1] Li Tianyi. (2015) Criticism of "Lousy Movies with High Box Office Receipts” Phenomenon of China's Current Films. Sichuan Drama, 02: 33-36.

[2] Zheng Yiyong. (2014) Phenomenon of Indulged Rotten Films. Arts Criticism, 05: 92-96.

[3] Wang Chujing. (2015) The Research of Lousy Movies with High Box-office Receipts and Marketing Strategy of Chinese Films. Changsha: Hunan Normal University

[4] Hu Yihao. (2013) The Surging Path of and Reflection on the Negative Review Marketing of Chinese Films[J]. Chinese Film Market, 11: 21-22.

[5] Yao Rui. (2011) Film Marketing in the Context of New Media: The Interactive Practice of Twitter and Film Marketing. Contemporary Cinema, 06: 134137.

[6] Liu Fan, Liu Jingya. (2014) On Key Issues of Film Marketing in China[J]. Journal of Shanghai University (Social Sciences), 31(05): 12-28.

[7] Su Xingsha. (2016) Image's Power: Hollywood Films and the Making and Dissemination of American Values Since 1990s. Changchun: Jilin University

[8] Hu Yun. (2014) The Commercial Strategies of Hollywood Films in the Context of Globalization. Modern Communication (Journal of Communication University of China). 36(06): 161 162.

[9] Hou Li Youmei. (2015) Rising Box Office, Degenerate Movies: On the "Post-modern" Image Spectacle of Current Chinese Films. J. Sichuan Drama, 02: 36-39.

[10] Zhang Weiping. (2003) New Picture Invests Films in the Pursuit of Refinement. Film Art, 03: 11-15.

[11] Hou Keming. (2021) The Creative Gains and Losses of Detective Chinatown 3. J. Film Art, 02: 92-94.

[12] Xiang Yong, Bai Xiaoqing. (2016) Field Resonance: A Cross-field Development Strategy for the IP Value of Internet Literature. J. Modern Communication, 08: 110-114. 